\title{
A subspace algorithm for simultaneous identification and input reconstruction
}

\author{
Harish J. Palanthandalam-Madapusi ${ }^{1}$ and Dennis S. Bernstein ${ }^{2, *, \dagger}$ \\ ${ }^{1}$ Department of Mechanical and Aerospace Engineering, Syracuse University, 149 Link Hall, \\ Syracuse, NY 13244, U.S.A. \\ ${ }^{2}$ Department of Aerospace Engineering, The University of Michigan, Ann Arbor, MI 48109, U.S.A.
}

\begin{abstract}
SUMMARY
This paper considers the concept of input and state observability, that is, conditions under which both the unknown input and initial state of a known model can be determined from output measurements. We provide necessary and sufficient conditions for input and state observability in discrete-time systems. Next, we develop a subspace identification algorithm that identifies the state-space matrices and reconstructs the unknown input using output measurements and known inputs. Finally, we present several illustrative examples, including a nonlinear system in which the unknown input is due to the endogenous nonlinearity. Copyright (c) 2008 John Wiley \& Sons, Ltd.
\end{abstract}

Received 12 April 2007; Revised 8 January 2008; Accepted 7 May 2008

KEY WORDS: subspace identification; input reconstruction; state estimation

\section{INTRODUCTION}

Systems with unknown inputs have received considerable attention [1-26]. The unknown inputs may represent unknown external drivers, input uncertainty, or instrument faults. An active research area is a state reconstruction with known model equations and unknown inputs. Approaches include full-order

\footnotetext{
*Correspondence to: Dennis S. Bernstein, Department of
Aerospace Engineering, The University of Michigan, Ann Arbor,

*Correspondence to: Dennis S. Bernstein, Department of
Aerospace Engineering, The University of Michigan, Ann Arbor, MI 48109, U.S.A. †E-mail: dsbaero@umich.edu, dsbaero@engin.umich.edu

Contract/grant sponsor: National Science Foundation Information Technology Research; contract/grant number: ATM-0325332

Copyright (C) 2008 John Wiley \& Sons, Ltd.
}

observers $[6,4,7,16,19,25]$, reduced-order observers $[1,5,12,13]$, geometric techniques [11], and trial-anderror methods [2]. A widely used approach is to model the unkno2n inputs as outputs of a known dynamic system and incorporate the input dynamics with the plant dynamics $[10,27]$. However, this approach increases the dimension of the observer and is limited to specific types of inputs.

In $[23,24]$ input reconstruction is achieved by inverting the known transfer function. More recently, methods for input reconstruction using optimal filters are developed in $[3,15,19,20,25]$. The methods of $[3,15,19,20,23-25]$ for state reconstruction and input reconstruction require knowledge of the model equations.

A related problem is the concept of input and state observability, which is the ability to reconstruct the 
inputs and states using only output measurements. Necessary and sufficient conditions for the input and state observability for continuous-time systems in terms of the invariant zeros of the system are presented in $[3,5,13,19,21]$. Input and state observability for discrete-time systems is considered in [3], whereas Floquet and Barbot [25] uses a constructive algorithm to determine the observability of the unknown input and state.

Subspace identification algorithms are used to identify systems in a state-space form, and are naturally applicable to multi-input, multi-output systems [28-39]. The idea underlying subspace algorithms is that estimates of the state sequence in an unknown basis can be computed directly from input-output observations. Once the state estimates are available, state-space matrices are estimated using least squares. These methods are computationally tractable and require no a priori information about the structure or order of the system.

In this paper, we examine conditions under which both the input and state can be estimated from the output measurements. We discuss necessary and sufficient conditions for a discrete-time system to be input and state observable and derive tests for input and state observability. Since no assumptions on the input are made, the unknown input can be either an unmodeled exogenous signal or a consequence of an unknown endogenous nonlinear function of the states.

We then develop a deterministic subspace identification algorithm for systems with arbitrary unknown inputs. When the conditions for input and state observability and persistency of excitation are satisfied, we show that the states, the state-space matrices, and the unknown inputs can be estimated from the output measurements. No assumptions are imposed on the unknown inputs.

Finally, we present several illustrative examples. For a linear example with a known model and an unknown exogenous input, we estimate the unknown input based on noisy output measurements. We then assume that the model is unknown and estimate both the model and the unknown input based on noisy output measurements. Furthermore, we consider a nonlinear system in which the unknown input is due to the endogenous nonlinearity.

\section{INPUT AND STATE OBSERVABILITY: STRICTLY PROPER CASE}

Consider the system

$$
\begin{gathered}
x_{k+1}=A x_{k}+H e_{k} \\
y_{k}=C x_{k}
\end{gathered}
$$

where $x_{k} \in \mathbb{R}^{n}, e_{k} \in \mathbb{R}^{p}, y_{k} \in \mathbb{R}^{l}, A \in \mathbb{R}^{n \times n}, H \in \mathbb{R}^{n \times p}$, and $C \in \mathbb{R}^{l \times n}$. Without loss of generality, we assume $l \leqslant n, \operatorname{rank}(C)=l>0$, and $\operatorname{rank}(H)=p>0$. No assumptions on the unmeasured signal $e_{k}$ are made. Hence, $e_{k}$ can be either an exogenous input or a consequence of nonlinear, time-varying function of the states.

Throughout this paper, $r$ denotes a nonnegative integer. Furthermore, for convenience, every vector or matrix with zero rows or zero columns is an empty matrix. Define $\mathscr{Y}_{r} \in \mathbb{R}^{(r+1) l}$ and $\mathscr{E}_{r} \in \mathbb{R}^{(r+1) p}$ as

$$
\mathscr{Y}_{r} \triangleq\left[\begin{array}{c}
y_{0} \\
y_{1} \\
\vdots \\
y_{r}
\end{array}\right], \quad \mathscr{E}_{r} \triangleq\left[\begin{array}{c}
e_{0} \\
e_{1} \\
\vdots \\
e_{r}
\end{array}\right]
$$

Definition 2.1

Let $r \geqslant 1$. Then the input and state unobservable subspace $\mathfrak{U}_{r}$ of (1), (2) is the subspace

$$
\mathfrak{U}_{r} \triangleq\left\{\left[\begin{array}{c}
x_{0} \\
\mathscr{E}_{r-1}
\end{array}\right] \in \mathbb{R}^{n+r p}: \mathscr{Y}_{r}=0\right\}
$$

We define $\Gamma_{r} \in \mathbb{R}^{(r+1) l \times n}, M_{r} \in \mathbb{R}^{(r+1) l \times r p}$, and $\Psi_{r} \in$ $\mathbb{R}^{(r+1) l \times(n+r p)}$ as

$$
\Gamma_{r} \triangleq\left[\begin{array}{c}
C \\
C A \\
C A^{2} \\
\vdots \\
C A^{r}
\end{array}\right]
$$

Int. J. Adapt. Control Signal Process. 2009; 23:1053-1069 DOI: $10.1002 /$ acs 


$$
M_{r} \triangleq\left[\begin{array}{cccc}
0 & 0 & \cdots & 0 \\
C H & 0 & \cdots & 0 \\
C A H & C H & \cdots & 0 \\
\vdots & \vdots & \ddots & \vdots \\
C A^{r-1} H & C A^{r-2} H & \cdots & C H
\end{array}\right]
$$

and

$$
\Psi_{r} \triangleq\left[\begin{array}{ll}
\Gamma_{r} & M_{r}
\end{array}\right]
$$

Note that $M_{0}$ is an empty matrix and thus $\Psi_{0}=\Gamma_{0}=C$. Next, from (1), (2), we can express

$$
\mathscr{Y}_{r}=\Gamma_{r} x_{0}+M_{r} \mathscr{E}_{r-1}=\Psi_{r}\left[\begin{array}{c}
x_{0} \\
\mathscr{E}_{r-1}
\end{array}\right]
$$

so that

$$
\mathfrak{U}_{r}=\mathscr{N}\left(\Psi_{r}\right)
$$

where $\mathscr{N}$ denotes the null space. Next, define the positive integer

$$
r_{0} \triangleq \begin{cases}\max \left\{\left\lceil\frac{n-l}{l-p}\right\rceil, 1\right\}, & p<l \\ 1, & p=l\end{cases}
$$

where $\lceil a\rceil$ denotes the smallest integer greater than or equal to $a$. Note that $r_{0}$ is not defined in the case $p>l$.

\section{Proposition 2.1}

Assume that $n \geqslant 2$ and $p \leqslant l$. Then $r_{0} \leqslant n-1$.

\section{Proof}

Suppose $p=l$, then $n-1 \geqslant 1=r_{0}$. Next, suppose $p<l$. If $\lceil(n-l) /(l-p)\rceil \leqslant 1$ then $n-1 \geqslant 1=r_{0}$. If $\lceil(n-l) /(l-p)\rceil>1$, then, since $n-1>n-l$ and $l-p \geqslant 1$, it follows that $r_{0}=\lceil(n-l) /(l-p)\rceil \leqslant\lceil n-$ $l\rceil \leqslant\lceil n-1\rceil=n-1$.

\section{Proposition 2.2}

Let $r \geqslant 1$. If $\mathfrak{U}_{r}=\{0\}$, then the following statements hold:

1. $p \leqslant l$.

2. If $p=l$, then $p=l=n$.

3. $(A, C)$ is observable, that is, $\operatorname{rank}\left(\Gamma_{n-1}\right)=n$.
4. $r \geqslant r_{0}$.

5. $\operatorname{rank}(C H)=p$.

6. $\operatorname{rank}\left(\Psi_{r}\right)=\operatorname{rank}\left(\Psi_{r-1}\right)+p$ for all $r \geqslant r_{0}$.

Proposition 2.3

Assume that either $p<l$ or $p=l=n$. Then $n+r p \leqslant$ $(r+1) l$ for all $r \geqslant r_{0}$.

\section{Proof}

Suppose $p=l=n$. Then $n+r p=(r+1) l$ for all $r>0$. Next, suppose $p<l$, let $r \geqslant r_{0}$, and assume $(r+1) l<n+$ $r p$ so that $r l-r p<n-l$. Hence $r<(n-l) /(l-p)$, and thus $\lceil(n-l) /(l-p)\rceil \leqslant r_{0}<(n-l) /(l-p)$, which is a contradiction. Thus, $n+r p \leqslant(r+1) l$.

Proposition 2.3 implies that if $p<l$ or $p=l=n$, then, for all $r \geqslant r_{0}$, the number of columns of $\Psi_{r}$ is less than or equal to the number of rows of $\Psi_{r}$.

\section{Definition 2.2}

System (1), (2) is input and state observable if $\mathfrak{U}_{r}=\{0\}$ for all $r \geqslant r_{0}$.

Definition 2.2 implies that if (1), (2) is input and state observable, then, for all $r \geqslant r_{0}$, the initial condition $x_{0}$ and input sequence $\left\{e_{i}\right\}_{i=0}^{r-1}$ are uniquely determined from the measured output sequence $\left\{y_{i}\right\}_{i=0}^{r}$.

\section{Theorem 2.1}

The following statements are equivalent:

1. System (1), (2) is input and state observable.

2. For all $r \geqslant r_{0}, \mathscr{Y}_{r}=0$ if and only if $\left[\begin{array}{c}x_{0} \\ \mathscr{E}_{r-1}\end{array}\right]=0$.

3. For all $r \geqslant r_{0}, \operatorname{rank}\left(\Psi_{r}\right)=n+r p$.

4. There exists $r \geqslant r_{0}$ such that $\operatorname{rank}\left(\Psi_{r}\right)=n+r p$.

5. $\operatorname{rank}\left(\Psi_{n-1}\right)=n+(n-1) p$.

\section{Proof}

From Definitions 2.1 and 2.2 it follows that $(1) \Rightarrow$ (2). Using (7), (2) $\Rightarrow(3)$. Result (3) $\Rightarrow$ (4) is immediate. To prove $(4) \Rightarrow(5)$ let $n=1$. Then $\Psi_{0}=C$ and $\operatorname{rank}(C)=1$. Now, suppose $n \geqslant 2$. Since $\operatorname{rank}\left(\Psi_{r}\right)=$ $n+r p$ it follows that $\operatorname{rank}(C H)=p$. Hence, for all $\hat{r} \geqslant r_{0}, \operatorname{rank}\left(\Psi_{\hat{r}}\right)=\operatorname{rank}\left(\Psi_{\hat{r}-1}\right)+p$. Hence, since $n-1 \geqslant r_{0}$, we have $\operatorname{rank}\left(\Psi_{n-1}\right)=n+(n-1) p$. Finally to show (5) $\Rightarrow(1)$, we consider two cases. First, suppose $n=1$. In this case, $C$ and $H$ are nonzero scalars, and hence it follows that $\operatorname{rank}\left(\Psi_{r}\right)=n+r p$ for all $r \geqslant r_{0}$

Int. J. Adapt. Control Signal Process. 2009; 23:1053-1069 
and hence $\mathfrak{U}_{r}=\{0\}$ for all $r \geqslant r_{0}$. Next, suppose $n \geqslant 2$. In this case, $\operatorname{rank}\left(\Psi_{n-1}\right)=n+(n-1) p$ implies that $\operatorname{rank}(C H)=p$ and hence $\operatorname{rank}\left(\Psi_{r}\right)=\operatorname{rank}\left(\Psi_{r-1}\right)+p$ for all $r \geqslant r_{0}$. Next, since $n-1 \geqslant r_{0}$, it follows that, for all $r \geqslant r_{0}, \operatorname{rank}\left(\Psi_{r}\right)=\operatorname{rank}\left(\Psi_{n-1}\right)+(r-n+1) p$. Thus, $\operatorname{rank}\left(\Psi_{r}\right)=n+r p$ for all $r \geqslant r_{0}$ and hence $\mathfrak{U}_{r}=\{0\}$ for all $r \geqslant r_{0}$.

Theorem 2.1 shows that (1), (2) is input and state observable if and only if $\Psi_{r}$ has full column rank for all $r \geqslant r_{0}$. In this case, the unique solution of (7) is

$$
\left[\begin{array}{c}
x_{0} \\
\mathscr{E}_{r-1}
\end{array}\right]=\Psi_{r}^{\dagger} \mathscr{Y}_{r}
$$

where $\dagger$ represents the Moore-Penrose generalized inverse $\Psi_{r}^{\dagger}=\left(\Psi_{r}^{\mathrm{T}} \Psi_{r}\right)^{-1} \Psi_{r}^{\mathrm{T}}$. Also, note that the system invertibility condition in Theorem 2 of [23] is closely related to the rank conditions 5 of Theorem 2.1.

Note that if no unknown inputs are present, that is, $p=0$, then $\Psi_{r}=\Gamma_{r}$, and statement 5 of Theorem 2.1 becomes the standard rank test for observability.

\section{INPUT AND STATE OBSERVABILITY: EXACTLY PROPER CASE}

Next, we consider the system

$$
\begin{gathered}
x_{k+1}=A x_{k}+H e_{k} \\
y_{k}=C x_{k}+G e_{k}
\end{gathered}
$$

where $G \in \mathbb{R}^{l \times p}$, whereas $A, H, C, x_{k}, e_{k}$, and $y_{k}$ are defined as in (1), (2). Without loss of generality, we assume $l \leqslant n, \operatorname{rank}(C)=l>0$, and $\operatorname{rank}\left[\begin{array}{c}H \\ G\end{array}\right]=p>0$. Due to $G e_{k}$, the output $y_{k}$ is directly affected by $e_{k}$ as well as by the past values of $e_{k}$. Therefore, we have

$$
\mathscr{Y}_{r}=\bar{\Psi}_{r}\left[\begin{array}{c}
x_{0} \\
\mathscr{E}_{r}
\end{array}\right]
$$

where $\mathscr{E}_{r} \quad$ is defined by (3), $\quad \bar{\Psi}_{r} \triangleq\left[\begin{array}{ll}\Gamma_{r} & \bar{M}_{r}\end{array}\right] \epsilon$ $\mathbb{R}^{(r+1) l \times[n+(r+1) p]}$, and

$$
\bar{M}_{r}=\left[\begin{array}{ccccc}
G & 0 & \cdots & 0 & 0 \\
C H & G & \cdots & 0 & 0 \\
\vdots & \vdots & \ddots & \vdots & \\
C A^{r-2} H & C A^{r-3} H & \cdots & G & 0 \\
C A^{r-1} H & C A^{r-2} H & \cdots & C H & G
\end{array}\right]
$$

Furthermore, we have the following definition.

Definition 3.1

Let $r \geqslant 0$. Then the input and state unobservable subspace $\overline{\mathfrak{U}}_{r}$ of (11), (12) is the subspace

$$
\overline{\mathfrak{U}}_{r} \triangleq\left\{\left[\begin{array}{c}
x_{0} \\
\mathscr{E}_{r}
\end{array}\right] \in \mathbb{R}^{n+(r+1) p}: \mathscr{Y}_{r}=0\right\}
$$

The input and state unobservable subspace is given by $\overline{\mathfrak{U}}_{r}=\mathscr{N}\left(\bar{\Psi}_{r}\right)$. Next, if $p<l$ then define

$$
\bar{r}_{0} \triangleq\left\lceil\frac{n}{l-p}\right\rceil-1
$$

Since $n>l-p$ it follows that $\bar{r}_{0} \geqslant 1$.

Proposition 3.1

Let $r \geqslant 0$. If $\overline{\mathfrak{U}}_{r}=\{0\}$, then the following statements hold:

1. $p<l$.

2. $n>1$.

3. $(A, C)$ is observable, that is, $\operatorname{rank}\left(\Gamma_{n-1}\right)=n$.

4. $r \geqslant \bar{r}_{0}$.

5. $\operatorname{rank}(G)=p$.

6. $\operatorname{rank}\left(\bar{\Psi}_{r}\right)=\operatorname{rank}\left(\bar{\Psi}_{r-1}\right)+p$ for all $r \geqslant \bar{r}_{0}$.

\section{Definition 3.2}

System (11), (12) is input and state observable if $\overline{\mathfrak{U}}_{r}=$ $\{0\}$ for all $r \geqslant \bar{r}_{0}$.

\section{Theorem 3.1}

The following statements are equivalent:

1. System (11), (12) is input and state observable.

2. For all $r \geqslant \bar{r}_{0}, \mathscr{Y}_{r}=0$ if and only if $\left[\begin{array}{c}x_{0} \\ \mathscr{E}_{r}\end{array}\right]=0$.

3. $\operatorname{rank}\left(\bar{\Psi}_{r}\right)=n+(r+1) p$ for all $r \geqslant \bar{r}_{0}$.

Int. J. Adapt. Control Signal Process. 2009; 23:1053-1069

DOI: $10.1002 / \mathrm{acs}$ 
4. There exists $r \geqslant \bar{r}_{0}$ such that $\operatorname{rank}\left(\bar{\Psi}_{r}\right)=n+$ $(r+1) p$.

5. $\operatorname{rank}\left(\bar{\Psi}_{n-1}\right)=n(p+1)$.

Finally, if (11), (12) is input and state observable, then Theorem 3.1 implies that $\bar{\Psi}_{r}$ has a full column rank for all $r \geqslant \bar{r}_{0}$. In this case, the unique solution of (13) is

$$
\left[\begin{array}{c}
x_{0} \\
\mathscr{E}_{r}
\end{array}\right]=\bar{\Psi}_{r}^{\dagger} \mathscr{Y}_{r}
$$

\section{NOISE ANALYSIS FOR INPUT AND STATE OBSERVABILITY}

To analyze the sensitivity of (10) to noise, consider (1), (2) with additive measurement and process noise so that

$$
\begin{gathered}
x_{k+1}=A x_{k}+H e_{k}+w_{k} \\
y_{k}=C x_{k}+v_{k}
\end{gathered}
$$

where $w_{k} \in \mathbb{R}^{n}$ and $v_{k} \in \mathbb{R}^{l}$ are zero mean, uncorrelated, white-noise sequences. Then

$$
\mathscr{Y}_{r}=\Psi_{r}\left[\begin{array}{c}
x_{0} \\
\mathscr{E}_{r-1}
\end{array}\right]+N_{r} \mathscr{W}_{r-1}+\mathscr{V}_{r}
$$

where

$$
\begin{gathered}
N_{r} \triangleq\left[\begin{array}{cccc}
0 & 0 & \cdots & 0 \\
C & 0 & \cdots & 0 \\
C A & C & \cdots & 0 \\
\vdots & \vdots & \ddots & \vdots \\
C A^{r-1} & C A^{r-2} & \cdots & C
\end{array}\right] \in \mathbb{R}^{(r+1) l \times r n} \\
\mathscr{W}_{r} \triangleq\left[\begin{array}{c}
w_{0} \\
w_{1} \\
\vdots \\
w_{r}
\end{array}\right] \in \mathbb{R}^{(r+1) n}, \quad \mathscr{V}_{r} \triangleq\left[\begin{array}{c}
v_{0} \\
v_{1} \\
\vdots \\
v_{r}
\end{array}\right] \in \mathbb{R}^{(r+1) l}
\end{gathered}
$$

Copyright (C) 2008 John Wiley \& Sons, Ltd.
We thus consider the least-squares estimate

$$
\left[\begin{array}{c}
\hat{x}_{0} \\
\hat{\mathscr{E}}_{r-1}
\end{array}\right] \triangleq \Psi_{r}^{\dagger} \mathscr{Y}_{r}=\left[\begin{array}{c}
x_{0} \\
\mathscr{E}_{r-1}
\end{array}\right]+\Psi_{r}^{\dagger} N_{r} \mathscr{W}_{r-1}+\Psi_{r}^{\dagger} \mathscr{V}_{r}
$$

Since $w_{k}$ and $v_{k}$ are zero mean noise sequences, (21) implies that

$$
\mathbb{E}\left[\begin{array}{c}
\hat{x}_{0} \\
\hat{\mathscr{E}}_{r-1}
\end{array}\right]=\left[\begin{array}{c}
x_{0} \\
\mathscr{E}_{r-1}
\end{array}\right]
$$

and thus (21) is an unbiased estimate of $\left[\begin{array}{c}x_{0} \\ \mathscr{E}_{r-1}\end{array}\right]$. Finally, the variance of estimate (21) is given by

$$
\operatorname{var}\left[\begin{array}{c}
\hat{x}_{0} \\
\hat{\mathscr{E}}_{r-1}
\end{array}\right]=\Psi_{r}^{\dagger} N_{r} R_{w} N_{r}^{\mathrm{T}}\left(\Psi_{r}^{\dagger}\right)^{\mathrm{T}}+\Psi_{r}^{\dagger} R_{v}\left(\Psi_{r}^{\dagger}\right)^{\mathrm{T}}
$$

where $R_{w} \triangleq \mathbb{E}\left[\begin{array}{ll}\mathscr{W}_{r-1} & \mathscr{W}_{r-1}^{\mathrm{T}}\end{array}\right]$ and $R_{v} \triangleq \mathbb{E}\left[\begin{array}{ll}\mathscr{V}_{r} & \mathscr{V}_{r}^{\mathrm{T}}\end{array}\right]$.

\section{COMPARTMENTAL MODEL EXAMPLE}

To illustrate the input and state observability with noisy data, we consider a system comprised $n=6$ compartments that exchange mass or energy through mutual interaction [40]. Applying conservation yields

$$
\begin{gathered}
x_{1, k+1}=x_{1, k}-\beta x_{1, k}+\alpha\left(x_{2, k}-x_{1, k}\right) \\
x_{i, k+1}=x_{i, k}-\beta x_{i, k}+\alpha\left(x_{i+1, k}-x_{i, k}\right) \\
\quad-\alpha\left(x_{i, k}-x_{i-1, k}\right), \quad i=2, \ldots, n-1 \\
x_{n, k+1}=x_{n, k}-\beta x_{n, k}-\alpha\left(x_{n, k}-x_{n-1, k}\right)
\end{gathered}
$$

where $0<\beta<1$ is the loss coefficient and $0<\alpha<1$ is the flow coefficient. In addition, an unknown input enters compartment 2 . The outputs are the energy states in compartments 2 and 3 , and therefore $l=2$ and $r_{0}=4$. It then follows that

$$
\begin{gathered}
x_{k+1}=A x_{k}+H e_{k} \\
y_{k}=C x_{k}
\end{gathered}
$$

where $A \in \mathbb{R}^{n \times n}, H \in \mathbb{R}^{n \times 1}$, and $C \in \mathbb{R}^{2 \times n}$ are defined as

$$
A \triangleq\left[\begin{array}{ccccc}
1-\beta-\alpha & \alpha & 0 & \cdots & 0 \\
\alpha & 1-\beta-\alpha & \alpha & \cdots & 0 \\
\vdots & & \ddots & \ddots & \vdots \\
0 & \cdots & 0 & \alpha & 1-\beta-\alpha
\end{array}\right],
$$

Int. J. Adapt. Control Signal Process. 2009; 23:1053-1069 DOI: $10.1002 / \mathrm{acs}$ 


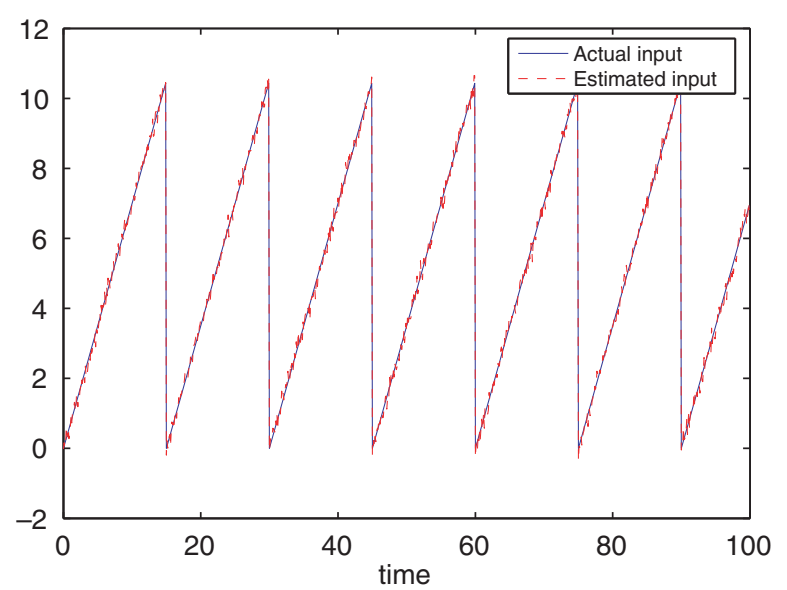

Figure 1. Compartmental model example. The actual unknown inputs and the estimates of the unknown inputs using measurements of outputs and the known model. Measurement and process noise with standard deviation 0.1 are added to the model simulation.

$$
\begin{gathered}
H \triangleq\left[\begin{array}{c}
0 \\
1 \\
\vdots \\
0
\end{array}\right] \\
C \triangleq\left[\begin{array}{lllll}
0 & 1 & 0 & \cdots & 0 \\
0 & 0 & 1 & \cdots & 0
\end{array}\right]
\end{gathered}
$$

For simulations, we set $\alpha=0.3$ and $\beta=0.1$. It can be verified that (27)-(30) is input and state observable.

The initial state is chosen to be $x_{0}=\left[\begin{array}{ll}2.0 & 0.1-\end{array}\right.$ $\left.\begin{array}{llll}1.0 & 0 & 0 & 0\end{array}\right]^{\mathrm{T}}$, and the unknown force is chosen to be a sawtooth signal. Simulations are run with the Gaussian process noise $w_{k}$ and measurement noise $v_{k}$ with covariances $\operatorname{diag}(0.01,0.01,0.01,0.01,0.01,0.01)$ and $\operatorname{diag}(0.01,0.01,0.01)$, respectively. Using the measured outputs, the initial state and unknown input are estimated using (10) for $r=1000$. Although (27)-(30) is input and state observable, poor numerical conditioning of $\Psi_{r}$ can cause the estimates of the unknown inputs to be inaccurate. In this example, the condition number of $\Psi_{r}$ is 82.8975 and thus $\Psi_{r}$ is not ill-conditioned. Figure 1 shows the unknown force and its estimate in the presence of process noise and measurement noise with standard deviation 0.1 . In the presence of process noise and measurement noise, the estimate of the initial state is $\hat{x}_{0}=\left[\begin{array}{llll}2.0690 & 0.1719 & -0.9862 & -\end{array}\right.$

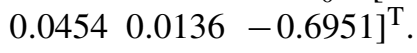

\section{CONNECTIONS WITH MULTIVARIABLE ZEROS}

In this section, we reinterpret the input and state observability conditions given by Theorem 2.1 for the strictly proper case in terms of multivariable transmission zeros.

For $\lambda \in \mathbb{C}$, define $v(\lambda) \in \mathbb{C}^{n-1}$ by

$$
v(\lambda)=\left[\begin{array}{lllll}
1 & \lambda & \lambda^{2} & \cdots & \lambda^{n-2}
\end{array}\right]^{\mathrm{T}}
$$

and $V(\lambda) \in \mathbb{C}^{[n+(n-1) p] \times(n+p)}$ by

$$
V(\lambda) \triangleq\left[\begin{array}{cc}
-I_{n} & 0 \\
0 & I_{p} \\
0 & \lambda I_{p} \\
\vdots & \vdots \\
0 & \lambda^{n-2} I_{p}
\end{array}\right]=\left[\begin{array}{cc}
-I_{n} & 0 \\
0 & v(\lambda) \otimes I_{p}
\end{array}\right]
$$

where $\otimes$ is the Kronecker product. Next, note that

$$
\Psi_{n-1} V(\lambda)=\left[\begin{array}{cc}
-C & 0 \\
-C A & C H \\
-C A^{2} & C A H+\lambda C H \\
\vdots & \vdots \\
-C A^{n-1} & C A^{n-2} H+\lambda C A^{n-3} H+\cdots+\lambda^{n-2} C H
\end{array}\right]
$$




\section{Lemma 6.1}

Let $\lambda_{1}, \lambda_{2}, \ldots, \lambda_{n-1} \in \mathbb{C}$ be distinct. Then

$$
\operatorname{rank}\left[\begin{array}{lll}
V\left(\lambda_{1}\right) & \cdots & V\left(\lambda_{n-1}\right)
\end{array}\right]=n+(n-1) p
$$

Proof

Note that

$$
\begin{aligned}
& \operatorname{rank}\left[\begin{array}{lll}
V\left(\lambda_{1}\right) & \cdots & V\left(\lambda_{n-1}\right)
\end{array}\right]
\end{aligned}
$$

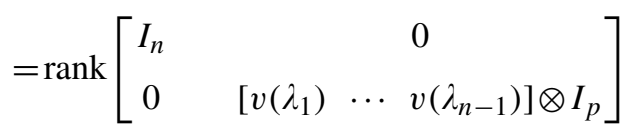

Next, since $\operatorname{rank}\left[v\left(\lambda_{1}\right) \cdots v\left(\lambda_{n-1}\right)\right]=n-1 \quad$ (Fact 5.13 .3 , p. 211 in [41]) and

$$
\begin{aligned}
& \operatorname{rank}\left[\left(\begin{array}{lll}
v\left(\lambda_{1}\right) & \cdots & v\left(\lambda_{n-1}\right)
\end{array}\right] \otimes I_{p}\right) \\
& =\left(\operatorname{rank}\left[\begin{array}{lll}
v\left(\lambda_{1}\right) & \cdots & v\left(\lambda_{n-1}\right)
\end{array}\right]\right) \operatorname{rank}\left(I_{p}\right) \\
& =(n-1) p
\end{aligned}
$$

it follows that

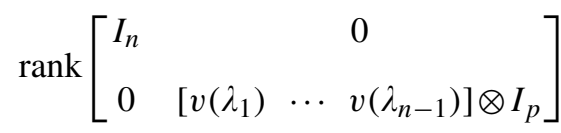

$$
\begin{aligned}
& =n+(n-1) p
\end{aligned}
$$

\section{Lemma 6.2}

Assume that $(A, C)$ is observable, $\operatorname{rank}\left(\Psi_{n-1} V(\lambda)\right)=$ $n+p$ for all $\lambda \in \mathbb{C}$, and either $p<l$ or $p=l=n$. Let $\lambda_{1}, \ldots, \lambda_{n-1} \in \mathbb{C}$ be distinct, then

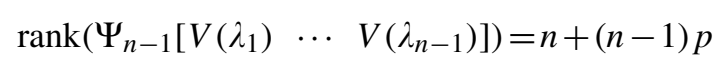

\section{Proof}

From Fact 2.10.24 in [41], we have

$$
\begin{aligned}
\operatorname{rank}( & \left.\Psi_{n-1}\left[V\left(\lambda_{1}\right) \quad V\left(\lambda_{2}\right)\right]\right) \\
= & \operatorname{rank}\left(\Psi_{n-1} V\left(\lambda_{1}\right)\right)+\operatorname{rank}\left(\Psi_{n-1} V\left(\lambda_{2}\right)\right) \\
& -\operatorname{dim}\left(\mathscr{R}\left(\Psi_{n-1} V\left(\lambda_{1}\right)\right) \cap \mathscr{R}\left(\Psi_{n-1} V\left(\lambda_{2}\right)\right)\right) \\
= & n+p+n+p \\
& -\operatorname{dim}\left(\Psi_{n-1} \mathscr{R}\left(V\left(\lambda_{1}\right)\right) \cap \Psi_{n-1} \mathscr{R}\left(V\left(\lambda_{2}\right)\right)\right)
\end{aligned}
$$

Copyright (C) 2008 John Wiley \& Sons, Ltd.

$$
\begin{aligned}
& =2(n+p)-\operatorname{dim}\left(\Psi_{n-1}\left[\mathscr{R}\left(V\left(\lambda_{1}\right)\right) \cap \mathscr{R}\left(V\left(\lambda_{2}\right)\right)\right]\right) \\
& =2(n+p)-n \\
& =n+2 p
\end{aligned}
$$

The penultimate identity follows from the fact that

$$
\mathscr{R}\left(V\left(\lambda_{1}\right)\right) \cap \mathscr{R}\left(V\left(\lambda_{2}\right)\right)=\mathscr{R}\left(\left[\begin{array}{c}
I_{n} \\
0 \\
\vdots \\
0
\end{array}\right]\right)
$$

and

$$
\operatorname{dim} \Psi_{n-1} \mathscr{R}\left(\left[\begin{array}{c}
I_{n} \\
0 \\
\vdots \\
0
\end{array}\right]\right)=\operatorname{dim} \mathscr{R}\left(\left[\begin{array}{c}
C \\
C A \\
\vdots \\
C A^{n-1}
\end{array}\right]\right)=n
$$

Next, let $2<k<n-1$ be an integer and assume that

$$
\operatorname{rank}\left(\Psi _ { n - 1 } \left[\begin{array}{llll}
V\left(\lambda_{1}\right) & V\left(\lambda_{2}\right) & \cdots & \left.\left.V\left(\lambda_{k}\right)\right]\right)=n+k p
\end{array}\right.\right.
$$

Next, we have

$$
\begin{aligned}
& \operatorname{rank}\left(\Psi_{n-1}\left[\begin{array}{lll}
V\left(\lambda_{1}\right) & \cdots & V\left(\lambda_{k+1}\right)
\end{array}\right)\right. \\
& =\operatorname{rank}\left(\Psi_{n-1}\left[\begin{array}{lll}
V\left(\lambda_{1}\right) & \cdots & V\left(\lambda_{k}\right)
\end{array}\right]\right) \\
& +\operatorname{rank}\left(\Psi_{n-1} V\left(\lambda_{k+1}\right)\right) \\
& -\operatorname{dim}\left(\mathscr { R } \left(\Psi_{n-1}\left[\begin{array}{llll}
V\left(\lambda_{1}\right) & \cdots & V\left(\lambda_{k}\right)
\end{array}\right)\right.\right. \\
& \left.\cap\left(\mathscr{R}\left(\Psi_{n-1} V\left(\lambda_{k+1}\right)\right)\right)\right)
\end{aligned}
$$

Next, since $p<l$ or $p=l=n$, it follows that

$$
\begin{gathered}
\operatorname{dim}\left(\mathscr{R}\left(\Psi_{n-1}\left[V\left(\lambda_{1}\right) \cdots V\left(\lambda_{k}\right)\right]\right)\right. \\
\left.\cap\left(\mathscr{R}\left(\Psi_{n-1} V\left(\lambda_{k+1}\right)\right)\right)\right)=n
\end{gathered}
$$

and thus

$$
\begin{aligned}
\operatorname{rank}\left(\Psi_{n-1}\left[\begin{array}{lll}
V\left(\lambda_{1}\right) & \cdots & \left.V\left(\lambda_{k+1}\right)\right]
\end{array}\right)\right. & =n+k p+n+p-n \\
& =n+(k+1) p
\end{aligned}
$$

Setting $k=n-2$ yields (36).

Int. J. Adapt. Control Signal Process. 2009; 23:1053-1069 DOI: $10.1002 / \mathrm{acs}$ 
Next, define the $l \times p$ rational transfer function matrix $L(z)$ by

$$
L(z) \triangleq C(z I-A)^{-1} H
$$

Furthermore, we assume that $(A, H, C)$ is minimal. Then $\lambda \in \mathbb{C}$ is an invariant zero of the realization $(A, H, C)$ if [42]

$$
\operatorname{rank}\left[\begin{array}{cc}
\lambda I-A & H \\
C & 0
\end{array}\right]<\operatorname{normalrank}\left[\begin{array}{cc}
z I-A & H \\
C & 0
\end{array}\right]
$$

Since $(A, H, C)$ is minimal, the transmission zeros of $L$ are the invariant zeros of $(A, H, C)$.

$$
\left[\begin{array}{cc}
0 & -I_{l} \\
C & 0 \\
C A & 0 \\
C A^{2} & 0 \\
\vdots & \\
C A^{n-1} & 0
\end{array}\right]\left[\begin{array}{c}
\lambda I-A \\
C
\end{array}\right.
$$

Lemma 6.3

The following statements are equivalent:

(i) normalrank $L=p$ and $L$ has no transmission zeros.

(ii) For all $\lambda \in \mathbb{C}$,

$$
\operatorname{rank}\left[\begin{array}{cc}
\lambda I-A & H \\
C & 0
\end{array}\right]=n+p
$$

Note that (ii) in Lemma 6.3 implies that (1)-(2) has no invariant zeros. The following result provides equivalent conditions for Theorem 2.1 in terms of multivariable zeros.

\section{Theorem 6.1}

The following statements are equivalent:

(i) Either $p<l$ or $p=l=n$, and $(A, H, C)$ has no invariant zeros.

(ii) $\operatorname{rank}\left(\Psi_{n-1}\right)=n+(n-1) p$.
Proof

To prove (i) $\Rightarrow$ (ii), it follows from (i) that, for all $\lambda \in \mathbb{C}$, $\operatorname{rank}\left[\begin{array}{c}\lambda I-A \\ C\end{array}\right]=n$, and thus $(A, C)$ is observable. Hence

$$
\operatorname{rank}\left[\begin{array}{cc}
0 & -I_{l} \\
C & 0 \\
C A & 0 \\
C A^{2} & 0 \\
\vdots & \\
C A^{n-1} & 0
\end{array}\right]=n+l
$$

Furthermore, noting that

$$
\left.\begin{array}{c}
H \\
0
\end{array}\right]=\left[\begin{array}{cc}
-C & 0 \\
\lambda C-C A & C H \\
\lambda C A-C A^{2} & C A H \\
\vdots & \\
\lambda C A^{n-2}-C A^{n-1} & C A^{n-2} H
\end{array}\right]
$$

it follows from Sylvester's inequality (Proposition 2.5.8 in [41]) that, for all $\lambda \in \mathbb{C}$,

$$
\begin{aligned}
n+\operatorname{rank}\left[\begin{array}{cc}
-C & 0 \\
\lambda C-C A & C H \\
\lambda C A-C A^{2} & C A H \\
\vdots & \vdots \\
\lambda C A^{n-2}-C A^{n-1} & C A^{n-2} H
\end{array}\right] \\
\geqslant \operatorname{rank}\left[\begin{array}{cc}
0 & -I_{l} \\
C & 0 \\
C A & 0 \\
C A^{2} & 0 \\
\vdots & \\
C A^{n-1} & 0
\end{array}\right]
\end{aligned}
$$

Int. J. Adapt. Control Signal Process. 2009; 23:1053-1069

DOI: $10.1002 / \mathrm{acs}$ 


$$
\begin{aligned}
& +\operatorname{rank}\left[\begin{array}{cc}
\lambda I-A & H \\
C & 0
\end{array}\right]-(n+l) \\
= & (n+l)+(n+p)-(n+l) \\
= & n+p
\end{aligned}
$$

Hence

$$
\operatorname{rank}\left[\begin{array}{cc}
-C & 0 \\
\lambda C-C A & C H \\
\lambda C A-C A^{2} & C A H \\
\vdots & \vdots \\
\lambda C A^{n-2}-C A^{n-1} & C A^{n-2} H
\end{array}\right]=n+p
$$

Next, for all $\lambda \in \mathbb{C}$, we have

$$
\begin{aligned}
& n+p=\operatorname{rank}\left[\begin{array}{cccc}
I_{n} & 0 & \cdots & 0 \\
\lambda I_{n} & I_{n} & \cdots & 0 \\
\vdots & \vdots & \ddots & \vdots \\
\lambda^{n-1} I_{n} & \lambda^{n-2} I_{n} & \cdots & I_{n}
\end{array}\right] \\
& \times {\left[\begin{array}{cc}
-C & 0 \\
\lambda C-C A & C H \\
\lambda C A-C A^{2} & C A H \\
\vdots & \\
\lambda C A^{n-2}-C A^{n-1} & C A^{n-2} H
\end{array}\right] }
\end{aligned}
$$

Next, using (33), (40) becomes

$$
\operatorname{rank}\left(\Psi_{n-1} V(\lambda)\right)=n+p
$$

Finally, let $\lambda_{1}, \lambda_{2}, \ldots, \lambda_{n-1} \in \mathbb{C}$ be distinct. Then, it follows from Lemma 6.2 and [41, Lemma 2.5.2] that

$$
\begin{aligned}
n+(n-1) p & =\operatorname{rank}\left(\Psi_{n-1}\left[\begin{array}{lll}
V\left(\lambda_{1}\right) & \cdots & V\left(\lambda_{n-1}\right)
\end{array}\right]\right) \\
& \leqslant \operatorname{rank}\left(\Psi_{n-1}\right)
\end{aligned}
$$

However, since $\operatorname{rank}\left(\Psi_{n-1}\right) \leqslant n+(n-1) p$, it follows that $\operatorname{rank}\left(\Psi_{n-1}\right)=n+(n-1) p$.
Next, to prove (ii) $\Rightarrow$ (i), suppose there exists $\lambda \in \mathbb{C}$ such that

$$
\operatorname{rank}\left[\begin{array}{cc}
\lambda I-A & H \\
C & 0
\end{array}\right]<n+p
$$

Then there exist $\tilde{x}_{0} \in \mathbb{C}^{n}$ and $\tilde{e} \in \mathbb{C}^{p}$ such that $\left[\begin{array}{c}\tilde{x}_{0} \\ \tilde{e}\end{array}\right]$ is nonzero and

$$
(\lambda I-A) \tilde{x}_{0}+H \tilde{e}=0
$$

and

$$
C \tilde{x}_{0}=0
$$

Premultiplying (41) by $C$ and using (42) yields

$$
-C A \tilde{x}_{0}+C H \tilde{e}=0
$$

Next, premultiplying (41) by $C A$ yields

$$
\lambda C A \tilde{x}_{0}-C A^{2} \tilde{x}_{0}+C A H \tilde{e}=0
$$

Using (43) in (44) yields

$$
-C A^{2} \tilde{x}_{0}+C A H \tilde{e}+\lambda C H \tilde{e}=0
$$

Similarly, premultiplying (41) by $C A^{2}, C A^{3}, \ldots$, $C A^{n-2}$ yields

$$
\begin{aligned}
& -C A^{3} \tilde{x}_{0}+C A^{2} H \tilde{e}+\lambda C A H \tilde{e}+\lambda^{2} C H \tilde{e}=0 \\
& -C A^{4} \tilde{x}_{0}+C A^{3} H \tilde{e}+\lambda C A^{2} H \tilde{e}+\lambda^{2} C A H \tilde{e}+\lambda^{3} C H \tilde{e} \\
& =0 \\
& \quad C \\
& \quad C A^{n-1} \tilde{x}_{0}+C A^{n-2} H \tilde{e}+\lambda C A^{n-3} H \tilde{e}+\cdots \\
& \quad+\lambda^{n-2} C H \tilde{e}=0
\end{aligned}
$$

Next, we express (43), (45)-(48) as

$$
\Psi_{n-1}\left[\begin{array}{c}
\tilde{x}_{0} \\
\tilde{\mathscr{E}}_{n-2}
\end{array}\right]=0
$$

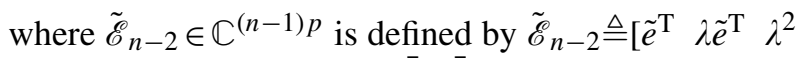
$\left.\begin{array}{lll}\tilde{e}^{\mathrm{T}} & \cdots & \lambda^{n-2} \tilde{e}^{\mathrm{T}}\end{array}\right]^{\mathrm{T}}$. Since $\left[\begin{array}{c}\tilde{x}_{0} \\ \tilde{e}\end{array}\right] \neq 0$, it follows that $\left[\begin{array}{c}\tilde{x}_{0} \\ \tilde{E}_{n-2}\end{array}\right] \neq 0$. However, $\operatorname{since} \operatorname{rank}\left(\Psi_{n-1}\right)=n+(n-1) p$, it follows that $\left[\begin{array}{c}\tilde{x}_{0} \\ \tilde{E}_{n-2}\end{array}\right]=0$, which contradicts $\left[\begin{array}{c}\tilde{x}_{0} \\ \tilde{E}_{n-2}\end{array}\right] \neq 0$.

Int. J. Adapt. Control Signal Process. 2009; 23:1053-1069 DOI: $10.1002 /$ acs 
Hence, $\operatorname{rank}\left[\begin{array}{cc}\lambda I-A & H \\ C & 0\end{array}\right]=n+p$ for all $\lambda \in \mathbb{C}$. Furthermore, using Proposition 2.2, it follows that either $p<l$ or $p=l=n$.

Note that (i) in the above result is same as the sufficient condition for input observability given in [5].

Next, define $\Phi_{r} \in \mathbb{R}^{(r+1) p \times(n+r l)}$ by

$$
\Phi_{r} \triangleq\left[\begin{array}{ccccc}
H & A H & A^{2} H & \cdots & A^{r} H \\
0 & C H & C A H & \cdots & C A^{r-1} H \\
0 & 0 & C H & \cdots & C A^{r-2} H \\
\vdots & \vdots & \vdots & \ddots & \vdots \\
0 & 0 & 0 & \cdots & C H
\end{array}\right]
$$

The following result is the dual of Theorem 6.1.

\section{Theorem 6.2}

The following conditions are equivalent:

(i) Either $l<p$ or $l=p=n$, and $(A, H, C)$ has no invariant zeros.

(ii) $\operatorname{rank}\left(\Phi_{n-1}\right)=n+(n-1) l$.

\section{STATE ESTIMATION WITH UNKNOWN INPUTS AND UNKNOWN DYNAMICS}

Consider the system

$$
\begin{gathered}
x_{k+1}=A x_{k}+B u_{k}+H e_{k} \\
y_{k}=C x_{k}+D u_{k}+G e_{k}
\end{gathered}
$$

where $x_{k}, y_{k}, e_{k}, A, C, H, G$ are as in Section 2, $u_{k} \in \mathbb{R}^{m}, B \in \mathbb{R}^{n \times m}$, and $D \in \mathbb{R}^{l \times m}$. Furthermore, $u_{k}$ is a known input, whereas $e_{k}$ is an unknown signal. System (51), (52) is input and state observable if it is input and state observable with $u_{k} \equiv 0$. We consider the problem of estimating the state sequence $\left\{x_{k}\right\}_{k=0}^{\infty}$ using measurements of inputs $u_{k}$ and outputs $y_{k}$, assuming that $A, B, C, D, H, G$, and $e_{k}$ are unknown. The problem of estimating $A, B, C, D, H, G$, and $e_{k}$ is considered in the following section. We assume that $(A, B)$ is controllable, $p \leqslant l$ is known, but the order $n$ of the system is unknown. In this section we assume that $G \neq 0$ so that (51), (52) corresponds to the exactly proper case (11), (12). The case $G=0$ is discussed later.

Let $N+1$ be the number of available measurements, and let $i$ be an integer such that $n \leqslant i$ and $2 i-1<N$. Define $U_{0 \mid 2 i-1} \in \mathbb{R}^{2 m i \times(N-2 i+2)}, \quad U_{\mathrm{p}} \in \mathbb{R}^{m i \times(N-2 i+2)}$, and $U_{\mathrm{f}} \in \mathbb{R}^{m i \times(N-2 i+2)}$ by

$$
\begin{aligned}
U_{0 \mid 2 i-1} \triangleq & \triangleq\left[\begin{array}{cccc}
u_{0} & u_{1} & \cdots & u_{N-2 i+1} \\
u_{1} & u_{2} & \cdots & u_{N-2 i+2} \\
\vdots & \vdots & \ddots & \vdots \\
u_{i-1} & u_{i} & \cdots & u_{N-i} \\
\hline u_{i} & u_{i+1} & \cdots & u_{N-i+1} \\
u_{i+1} & u_{i+2} & \cdots & u_{N-i+2} \\
\vdots & \vdots & \ddots & \vdots \\
u_{2 i-1} & u_{2 i} & \cdots & u_{N}
\end{array}\right] \\
= & {\left[\frac{U_{0 \mid i-1}}{U_{i \mid 2 i-1}}\right]=\left[\frac{U_{\mathrm{p}}}{U_{\mathrm{f}}}\right] }
\end{aligned}
$$

Partitioning $U_{0 \mid 2 i-1}$ differently, we have

$$
\begin{aligned}
U_{0 \mid 2 i-1} & =\left[\begin{array}{cccc}
u_{0} & u_{1} & \cdots & u_{N-2 i+1} \\
u_{1} & u_{2} & \cdots & u_{N-2 i+2} \\
\vdots & \vdots & \ddots & \vdots \\
u_{i-1} & u_{i} & \cdots & u_{N-i} \\
u_{i} & u_{i+1} & \cdots & u_{N-i+1} \\
\hline u_{i+1} & u_{i+2} & \cdots & u_{N-i+2} \\
\vdots & \vdots & \ddots & \vdots \\
u_{2 i-1} & u_{2 i} & \cdots & u_{N}
\end{array}\right] \\
= & {\left[\begin{array}{cc}
U_{0 \mid i} \\
\frac{U_{i+1 \mid 2 i-1}}{i+1}
\end{array}\right]=\left[\begin{array}{c}
U_{\mathrm{p}}^{+} \\
U_{\mathrm{f}}^{-}
\end{array}\right] }
\end{aligned}
$$

where $U_{\mathrm{p}}^{+} \in \mathbb{R}^{(i+1) m \times(N-2 i+2)}$ and $U_{\mathrm{f}}^{-} \in \mathbb{R}^{(i-1) m \times(N-2 i+2)}$. The subscript $\mathrm{p}$ denotes 'past' and the subscript $\mathrm{f}$ denotes 'future'. The output block-Hankel matrices $Y_{0 \mid 2 i-1}, Y_{\mathrm{p}}, Y_{\mathrm{f}}, Y_{\mathrm{p}}^{+}$and $Y_{\mathrm{f}}^{-}$are defined as in (53)-(56) with $u$ replaced by $y$. The unknown-input block-Hankel matrices $E_{0 \mid 2 i-1}, E_{\mathrm{p}}, E_{\mathrm{f}}, E_{\mathrm{p}}^{+}$, and $E_{\mathrm{f}}^{-}$are defined as in (53)-(56) with $u$ replaced by $e$. Furthermore, define the past input-output data

$$
W_{\mathrm{p}} \triangleq\left[\begin{array}{c}
U_{\mathrm{p}} \\
Y_{\mathrm{p}}
\end{array}\right] \in \mathbb{R}^{i(m+l) \times(N-2 i+2)}
$$

Int. J. Adapt. Control Signal Process. 2009; 23:1053-1069 DOI: $10.1002 /$ acs 
and the future input-output data

$$
W_{\mathrm{f}} \triangleq\left[\begin{array}{c}
U_{\mathrm{f}} \\
Y_{\mathrm{f}}
\end{array}\right] \in \mathbb{R}^{i(m+l) \times(N-2 i+2)}
$$

Finally, define the block-Toeplitz matrix $\Omega_{i} \in$ $\mathbb{R}^{(i+1) l \times(i+1) m}$ by

$$
\Omega_{i} \triangleq\left[\begin{array}{ccccc}
D & 0 & \cdots & 0 & 0 \\
C B & D & \cdots & 0 & 0 \\
C A B & C B & \cdots & 0 & 0 \\
\vdots & \vdots & \ddots & \vdots & \vdots \\
C A^{i-1} B & C A^{i-2} B & \cdots & C B & D
\end{array}\right]
$$

and, for $0 \leqslant r \leqslant 2 i$, define the state sequence $X_{r} \in$ $\mathbb{R}^{n \times(N-2 i+2)}$ by

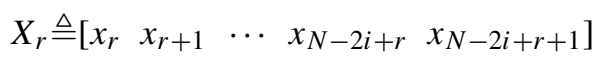

\section{Lemma 7.1}

If (51), (52) is input and state observable, then the row space of $X_{i}$ is contained in the intersection of the row space of $W_{\mathrm{p}}$ and the row space of $W_{\mathrm{f}}$.

\section{Proof}

From (51) and (52),

$$
\begin{gathered}
Y_{\mathrm{p}}=\Gamma_{i-1} X_{0}+\bar{M}_{i-1} E_{\mathrm{p}}+\Omega_{i-1} U_{\mathrm{p}} \\
Y_{\mathrm{f}}=\Gamma_{i-1} X_{i}+\bar{M}_{i-1} E_{\mathrm{f}}+\Omega_{i-1} U_{\mathrm{f}}
\end{gathered}
$$

Since the system is input and state observable, (60) can be expressed as

$$
\left[\begin{array}{c}
X_{i} \\
E_{\mathrm{f}}
\end{array}\right]=\left[\begin{array}{ll}
-\bar{\Psi}_{i-1}^{\dagger} \Omega_{i-1} & \bar{\Psi}_{i-1}^{\dagger}
\end{array}\right] W_{\mathrm{f}}
$$

Furthermore,

$$
X_{i}=\left[-\bar{\Psi}_{i-1, n}^{\dagger} \Omega_{i-1} \quad \bar{\Psi}_{i-1, n}^{\dagger}\right] W_{\mathrm{f}}
$$

where $\bar{\Psi}_{i-1, n}^{\dagger}$ denotes the first $n$ rows of $\bar{\Psi}_{i-1}^{\dagger}$. From (62), it follows that the state sequence $X_{i}$ is contained in the row space of $W_{\mathrm{f}}$. Next, we can relate $X_{0}$ and $X_{i}$ as

$$
X_{i}=A^{i} X_{0}+\Theta_{i} E_{\mathrm{p}}+\Delta_{i} U_{\mathrm{p}}
$$

Copyright (C) 2008 John Wiley \& Sons, Ltd. where

$$
\begin{gathered}
\Delta_{i} \triangleq\left[\begin{array}{llll}
A^{i-1} B & A^{i-2} B & \cdots & B
\end{array}\right] \\
\Theta_{i} \triangleq\left[\begin{array}{llll}
A^{i-1} H & A^{i-2} H & \cdots & H
\end{array}\right]
\end{gathered}
$$

Using (59) and (63), we obtain

$$
\begin{aligned}
X_{i} & =\left[\begin{array}{ll}
A^{i} & \Theta_{i}
\end{array}\right] \bar{\Psi}_{i-1}^{\dagger}\left(Y_{\mathrm{p}}-\Omega_{i-1} U_{\mathrm{p}}\right)+\Delta_{i} U_{\mathrm{p}} \\
& =\left[\begin{array}{ll}
\mathscr{A}_{1, i} & \mathscr{A}_{2, i}
\end{array}\right] W_{\mathrm{p}}
\end{aligned}
$$

where $\quad \mathscr{A}_{1, i} \triangleq-\left[\begin{array}{ll}A^{i} & \Theta_{i}\end{array}\right] \bar{\Psi}_{i-1}^{\dagger} \Omega_{i-1}+\Delta_{i} \quad$ and $\quad \mathscr{A}_{2, i}$ $\triangleq\left[\begin{array}{ll}A^{i} & \Theta_{i}\end{array}\right] \bar{\Psi}_{i}^{\dagger}$. From (65), the state sequence $X_{i}$ is also contained in the row space of $W_{\mathrm{p}}$. Thus, from (62) and (65) it follows that the state sequence $X_{i}$ is contained in the intersection of the row space of $W_{\mathrm{p}}$ and the row space of $W_{\mathrm{f}}$.

To calculate the state sequence, we require the following definition concerning

$$
\left[\begin{array}{c}
X_{0} \\
E_{0 \mid 2 i-1} \\
U_{0 \mid 2 i-1}
\end{array}\right] \in \mathbb{R}^{(n+2 p i+2 m i) \times(N-2 i+2)}
$$

\section{Definition 7.1}

The sequences $\left\{u_{k}\right\}_{k=1}^{N}$ and $\left\{e_{k}\right\}_{k=1}^{N}$ are persistently exciting for (51), (52) if

$$
\operatorname{rank}\left[\begin{array}{c}
X_{0} \\
E_{0 \mid 2 i-1} \\
U_{0 \mid 2 i-1}
\end{array}\right]=n+2 p i+2 m i
$$

If $\left\{u_{k}\right\}_{k=1}^{N}$ and $\left\{e_{k}\right\}_{k=1}^{N}$ are persistently exciting, then it follows from (66) that $X_{0}$ has full row rank, $\left[\begin{array}{l}E_{0 \mid 2 i-1} \\ U_{0 \mid 2 i-1}\end{array}\right]$ has full row rank, and the intersection of the row spaces of $X_{0}$ and $\left[\begin{array}{l}E_{0 \mid 2 i-1} \\ U_{0 \mid 2 i-1}\end{array}\right]$ is zero.

Theorem 7.1

If system (51), (52) is input and state observable and the sequences $\left\{u_{k}\right\}_{k=1}^{N}$ and $\left\{e_{k}\right\}_{k=1}^{N}$ are persistently exciting, then the intersection of the row spaces of $W_{\mathrm{p}}$ and $W_{\mathrm{f}}$ is equal to the row space of $X_{i}$.

Int. J. Adapt. Control Signal Process. 2009; 23:1053-1069 DOI: $10.1002 / \mathrm{acs}$ 


\section{Proof}

From Lemma 7.1, it follows that the intersection of the row spaces of $W_{\mathrm{p}}$ and $W_{\mathrm{f}}$ contains the state sequence $X_{i}$. Now, to show that the intersection of the row spaces of $W_{\mathrm{p}}$ and $W_{\mathrm{f}}$ is the row space of $X_{i}$, we show that the dimension of the intersection of the row spaces of $W_{\mathrm{p}}$ and $W_{\mathrm{f}}$ is $n$. Using (59) we express

$$
\left[\begin{array}{c}
Y_{\mathrm{p}} \\
U_{\mathrm{p}}
\end{array}\right]=\left[\begin{array}{ccc}
\Gamma_{i-1} & \bar{M}_{i-1} & \Omega_{i-1} \\
0 & 0 & I_{m i}
\end{array}\right]\left[\begin{array}{c}
X_{0} \\
E_{\mathrm{p}} \\
U_{\mathrm{p}}
\end{array}\right]
$$

Next, since (51), (52) is input and state observable and $\bar{r}_{0}<n<i$, it follows from Theorem 3.1 that $\operatorname{rank}\left(\bar{\Psi}_{i-1}\right)=\operatorname{rank}\left[\Gamma_{i-1} \bar{M}_{i-1}\right]=n+p i$, which implies that rank

$$
\left[\begin{array}{ccc}
\Gamma_{i-1} & \bar{M}_{i-1} & \Omega_{i-1} \\
0 & 0 & I_{m i}
\end{array}\right]=n+p i+m i
$$

and $l i+m i \leqslant n+p i+m i$. Therefore, it follows from (67) that

$$
\operatorname{rank}\left[\begin{array}{c}
Y_{\mathrm{p}} \\
U_{\mathrm{p}}
\end{array}\right]=\operatorname{rank}\left[\begin{array}{c}
X_{0} \\
E_{\mathrm{p}} \\
U_{\mathrm{p}}
\end{array}\right]=n+p i+m i
$$

Similarly,

$$
\operatorname{rank}\left[\begin{array}{c}
Y_{\mathrm{f}} \\
U_{\mathrm{f}}
\end{array}\right]=\operatorname{rank}\left[\begin{array}{c}
X_{i} \\
E_{\mathrm{f}} \\
U_{\mathrm{f}}
\end{array}\right]
$$

From (63) it follows that

$$
X_{i}=\left[\begin{array}{lll}
A^{i} & \Theta_{i} & \Delta_{i}
\end{array}\right]\left[\begin{array}{c}
X_{0} \\
E_{\mathrm{p}} \\
U_{\mathrm{p}}
\end{array}\right]
$$

Since $\operatorname{rank}\left[\begin{array}{lll}A^{i} & \Theta_{i} & \Delta_{i}\end{array}\right]=n$, it follows from Sylvester's inequality (Corollary 2.5.9 in [41]) that

$$
\operatorname{rank}\left(X_{i}\right)=n
$$

Copyright (c) 2008 John Wiley \& Sons, Ltd.
Finally, from (69), (70), and (66), we have

$$
\operatorname{rank}\left[\begin{array}{c}
Y_{\mathrm{f}} \\
U_{\mathrm{f}}
\end{array}\right]=n+p i+m i
$$

By similar arguments,

$$
\begin{aligned}
\operatorname{rank}\left[\begin{array}{c}
U_{\mathrm{p}} \\
Y_{\mathrm{p}} \\
U_{\mathrm{f}} \\
Y_{\mathrm{f}}
\end{array}\right] & =\operatorname{rank}\left[\begin{array}{c}
Y_{0 \mid 2 i-1} \\
U_{0 \mid 2 i-1}
\end{array}\right]=\operatorname{rank}\left[\begin{array}{c}
X_{0} \\
E_{0 \mid 2 i-2} \\
U_{0 \mid 2 i-1}
\end{array}\right] \\
& =n+2 p i+2 m i
\end{aligned}
$$

Now, the Grassmann dimension theorem [41, Theorem 2.3.1] gives

$$
\begin{aligned}
& \operatorname{dim}\left(\operatorname{row} \text { space }\left[\begin{array}{c}
U_{\mathrm{p}} \\
Y_{\mathrm{p}}
\end{array}\right] \cap \text { row space }\left[\begin{array}{c}
U_{\mathrm{f}} \\
Y_{\mathrm{f}}
\end{array}\right]\right) \\
& =\operatorname{rank}\left[\begin{array}{c}
U_{\mathrm{p}} \\
Y_{\mathrm{p}}
\end{array}\right]+\operatorname{rank}\left[\begin{array}{c}
U_{\mathrm{f}} \\
Y_{\mathrm{f}}
\end{array}\right]-\operatorname{rank}\left[\begin{array}{c}
U_{\mathrm{p}} \\
Y_{\mathrm{p}} \\
U_{\mathrm{f}} \\
Y_{\mathrm{f}}
\end{array}\right] \\
& =[m i+n+p i]+[m i+n+p i]-[2 m i+n+2 p i] \\
& =n
\end{aligned}
$$

The proofs of the above results are extensions of the proofs in $[38,39]$, with modifications in several key steps to address input reconstruction.

Let $\hat{X}_{i}$ denote an estimate of the state sequence $X_{i}$. Using Theorem 7.1, we compute $\hat{X}_{i}$ as the intersection of the row spaces of $W_{\mathrm{p}}$ and $W_{\mathrm{f}}$. One way to compute this intersection is by orthogonally projecting the row space of $W_{\mathrm{p}}$ onto the row space of $W_{\mathrm{f}}$ [28]. Thus

$$
\hat{X}_{i} \triangleq W_{\mathrm{f}} W_{\mathrm{p}}^{\mathrm{T}}\left(W_{\mathrm{p}} W_{\mathrm{p}}^{\mathrm{T}}\right)^{\dagger} W_{\mathrm{p}}
$$

Note that, to calculate $\hat{X}_{i}$, we use measurements of $u_{k}$ and $y_{k}$; however, knowledge of $e_{k}$ is not required.

Int. J. Adapt. Control Signal Process. 2009; 23:1053-1069 DOI: $10.1002 /$ acs 
A numerically efficient way to compute $\hat{X}_{i}$ is to use the LQ decomposition of $\left[\begin{array}{l}W_{\mathrm{p}} \\ W_{\mathrm{f}}\end{array}\right][28]$ given by

$$
\left[\begin{array}{c}
W_{\mathrm{p}} \\
W_{\mathrm{f}}
\end{array}\right]=L Q^{\mathrm{T}}=\left[\begin{array}{cc}
L_{11} & 0 \\
L_{21} & L_{22}
\end{array}\right]\left[\begin{array}{c}
Q_{1}^{\mathrm{T}} \\
Q_{2}^{\mathrm{T}}
\end{array}\right]
$$

where $L \in \mathbb{R}^{2 i(m+l) \times 2 i(m+l)}$ is lower triangular, $L_{11}, L_{21}$, $L_{22} \in \mathbb{R}^{i(m+l) \times i(m+l)}, Q \in \mathbb{R}^{(N-2 i+2) \times 2 i(m+l)}$ is orthogonal, and $Q_{1}, Q_{2} \in \mathbb{R}^{(N-2 i+2) \times i(m+l)}$. Then, the intersection of row spaces of $W_{\mathrm{p}}$ and $W_{\mathrm{f}}$ is computed as $L_{21} Q_{1}^{\mathrm{T}}$. An estimate $\hat{X}_{i}$ of the state sequence $X_{i}$ can then be obtained by using a singular value decomposition to calculate a basis for the row space of $L_{21} Q_{1}^{\mathrm{T}}$. Similarly, estimates $\hat{X}_{i+1}$ of the state sequence $X_{i+1}$ are obtained by computing the intersection of the row spaces of

$$
\left[\begin{array}{c}
U_{\mathrm{p}}^{+} \\
Y_{\mathrm{p}}^{+}
\end{array}\right] \text {and }\left[\begin{array}{c}
U_{\mathrm{f}}^{-} \\
Y_{\mathrm{f}}^{-}
\end{array}\right]
$$

Next, assume $G=0$ in (51), (52), which corresponds to the strictly proper case. The following result considers state estimation with unknown inputs and unknown dynamics.

\section{Theorem 7.2}

Assume that (51) and (52) with $G=0$ is input and state observable. If the input sequences $\left\{u_{k}\right\}_{k=1}^{N}$ and $\left\{e_{k}\right\}_{k=1}^{N}$ are persistently exciting, then the intersection of the row spaces of $\left[\begin{array}{c}U_{\mathrm{p}} \\ Y_{\mathrm{p}}^{+}\end{array}\right]$and $\left[\begin{array}{c}U_{\mathrm{f}} \\ Y_{\mathrm{f}}\end{array}\right]$ is the row space of $X_{i}$.

\section{Proof}

When $G=0$, the equations relating the input blockHankel matrices and the output block-Hankel matrices are given by

$$
\begin{gathered}
Y_{\mathrm{p}}^{+}=\Gamma_{i} X_{0}+M_{i} E_{\mathrm{p}}+\Omega_{i} U_{\mathrm{p}}^{+} \\
Y_{\mathrm{f}}=\Gamma_{i-1} X_{i}+M_{i-1} E_{i \mid 2 i-2}+\Omega_{i-1} U_{\mathrm{f}}
\end{gathered}
$$

Using (76) and (77) in place of (59) and (60) and following the steps of the proofs of Lemma 7.1, it follows that the state sequence $X_{i}$ is contained in the intersection of the row spaces of $\left[\begin{array}{c}U_{\mathrm{p}}^{+} \\ Y_{\mathrm{p}}^{+}\end{array}\right]$and $\left[\begin{array}{c}U_{\mathrm{f}} \\ Y_{\mathrm{f}}\end{array}\right]$. Furthermore, it follows from (53)-(56) that $U_{i \mid i}$ is contained in the intersection of the row spaces of $\left[\begin{array}{c}U_{\mathrm{p}}^{+} \\ Y_{\mathrm{p}}^{+}\end{array}\right]$and $\left[\begin{array}{c}U_{\mathrm{f}} \\ Y_{\mathrm{f}}\end{array}\right]$. Next, using arguments similar to the proof of 7.1 yields that the row space of $\left[\begin{array}{c}X_{i} \\ U_{i \mid i}\end{array}\right]$ is equal to the intersection of the row spaces of $\left[\begin{array}{c}U_{\mathrm{p}}^{+} \\ Y_{\mathrm{p}}^{+}\end{array}\right]$and $\left[\begin{array}{c}U_{\mathrm{f}} \\ Y_{\mathrm{f}}\end{array}\right]$. Thus, it follows that the row space of $X_{i}$ is the intersection of the row spaces of $\left[\begin{array}{c}U_{\mathrm{p}} \\ Y_{\mathrm{p}}^{+}\end{array}\right]$and $\left[\begin{array}{c}U_{\mathrm{f}} \\ Y_{\mathrm{f}}\end{array}\right]$ is the row space of $X_{i}$.

\section{SIMULTANEOUS MODEL ESTIMATION AND INPUT RECONSTRUCTION}

In this section we consider the problem of estimating the state-space matrices $A, B, C, D, H, G$, and $e_{k}$ of (51), (52) using estimates $\hat{X}_{i}$ of the state sequence $X_{i}$ and measurements of $u_{k}$ and $y_{k}$. To do this we express

$$
\left[\begin{array}{c}
X_{i+1} \\
Y_{i \mid i}
\end{array}\right]=\left[\begin{array}{ll}
A & B \\
C & D
\end{array}\right]\left[\begin{array}{c}
X_{i} \\
U_{i \mid i}
\end{array}\right]+\left[\begin{array}{c}
H \\
G
\end{array}\right] E_{i \mid i}
$$

We use a two-step procedure to estimate $A, B, C, D, H$, and $G$. First, we estimate the matrices $A, B, C$, and $D$ by solving the least-squares problem

$$
\underset{A, B, C, D}{\operatorname{argmin}}\left\|\left[\begin{array}{c}
\hat{X}_{i+1} \\
Y_{i \mid i}
\end{array}\right]-\left[\begin{array}{cc}
A & B \\
C & D
\end{array}\right]\left[\begin{array}{c}
\hat{X}_{i} \\
U_{i \mid i}
\end{array}\right]\right\|_{2}
$$

Although $\left[\begin{array}{c}X_{i+1} \\ Y_{i \mid i}\end{array}\right]$ is a linear combination of $\left[\begin{array}{c}X_{i} \\ U_{i \mid i}\end{array}\right]$ and $E_{i \mid i}$, the term due to $E_{i \mid i}$ is ignored in the least-squares problem (78). Thus, $E_{i \mid i}$ is interpreted as noise, and hence unbiased estimates of the state-space matrices are not guaranteed. However, if $\left[\begin{array}{l}x_{k} \\ u_{k}\end{array}\right]$ and $e_{k}$ are uncorrelated then unbiased estimates of $A, B, C$, and $D$ are obtained using (78). Next, defining the residual

$$
R_{i \mid i}=\left[\begin{array}{c}
\hat{X}_{i+1} \\
Y_{i \mid i}
\end{array}\right]-\left[\begin{array}{cc}
\hat{A} & \hat{B} \\
\hat{C} & \hat{D}
\end{array}\right]\left[\begin{array}{c}
\hat{X}_{i} \\
U_{i \mid i}
\end{array}\right]
$$


we estimate $\left[\begin{array}{c}H \\ G\end{array}\right]$ and $E_{i \mid i}$ by forming the singular value

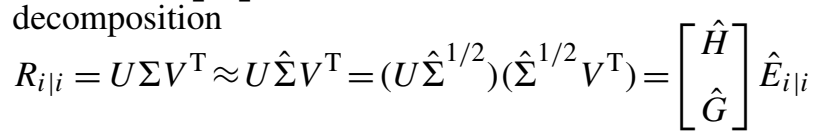

where $\hat{\Sigma}$ contains the $p$ dominant singular values from $\Sigma$, with

$$
\left[\begin{array}{c}
\hat{H} \\
\hat{G}
\end{array}\right] \triangleq U \hat{\Sigma}^{1 / 2} \text { and } \quad \hat{E}_{i \mid i} \triangleq \hat{\Sigma}^{1 / 2} V^{\mathrm{T}}
$$

Finally, consider the case in which $e_{k}$ is a nonlinear function of the states, that is, $e_{k}=h\left(x_{k}\right)$, where $h: \mathbb{R}^{n} \rightarrow$ $\mathbb{R}^{p}$. We assume that $h\left(x_{k}\right)$ can be expanded in terms of basis functions as

$$
h\left(x_{k}\right)=\theta f_{h}\left(x_{k}\right)
$$

where $f_{h}: \mathbb{R}^{n} \rightarrow \mathbb{R}^{s}$ are basis functions, and $\theta \in \mathbb{R}^{p \times s}$ are unknown coefficients of the basis function expansion. We thus estimate $\theta$ by solving the least-squares problem

$$
\underset{\theta}{\operatorname{argmin}}\left\|\hat{E}_{i \mid i}-\theta f_{h}\left(\hat{X}_{i}\right)\right\|_{2}
$$

When noise terms are present in (51) and (52) the states are estimated by obliquely projecting the row space of $Y_{\mathrm{f}}$ along the row space of $U_{\mathrm{f}}$ into the row space of $W_{\mathrm{p}}$ similar to the procedure presented in [28]. The least-squares problems for calculating the state-space matrices remain the same as (78), (80), and (82).

\section{COMPARTMENTAL MODEL EXAMPLE REVISITED}

We reconsider the compartmental model example as described in Section 5. In addition to the unknown input, we assume that the model is unknown and that a known input enters compartment 1 . Thus, $B \in \mathbb{R}^{n \times 1}$ is defined as

$$
B \triangleq\left[\begin{array}{llll}
1 & 0 & \cdots & 0
\end{array}\right]^{\mathrm{T}}
$$

To generate data for identification, we corrupt the system equations with process noise and measurement noise with standard deviation 0.1 . We take the known input to be a realization of a white-noise process, whereas the unknown input is a realization of a white-noise process with impulses at times 20 and 80 .

A comparison of the actual output 1 of the system and output 1 of the identified model is shown in Figures 2 and 3. Figure 4 shows the actual unknown input and the reconstructed unknown input is shown.

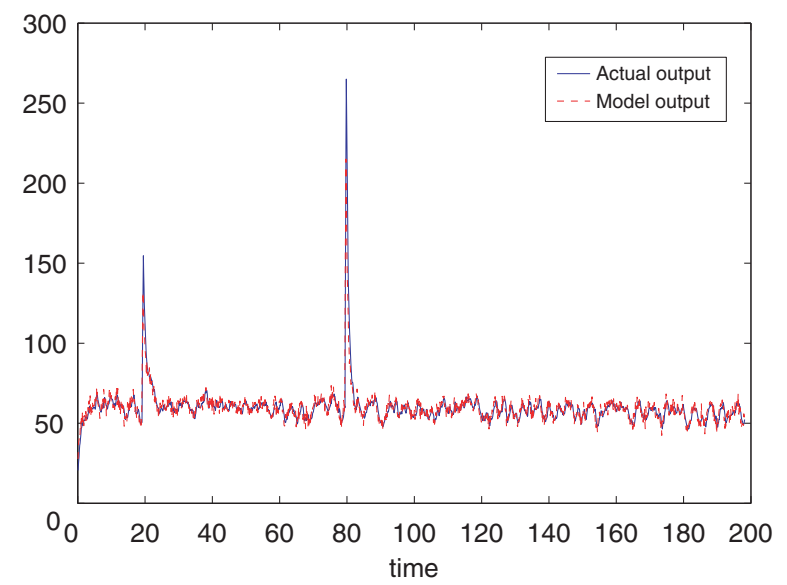

Figure 2. Compartmental model example. The actual energy of compartment 2 as well as the estimated energy of compartment 2 as determined by the identified model are shown.

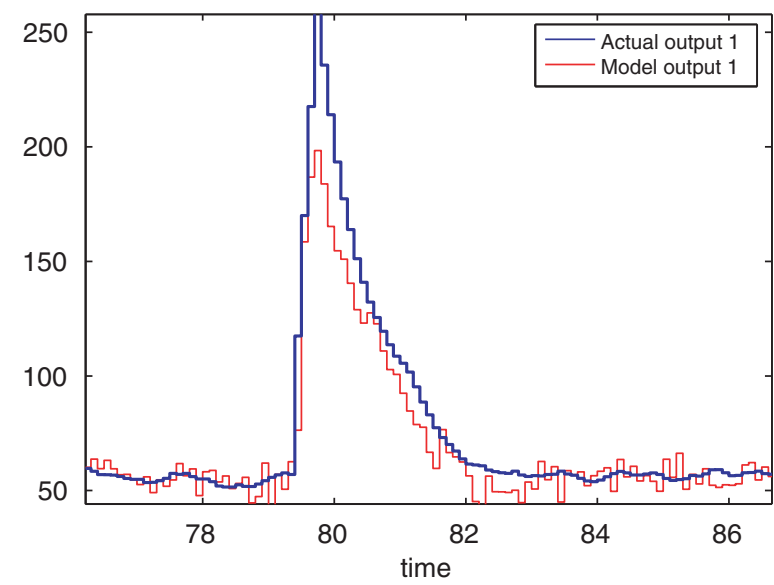

Figure 3. Compartmental model example. The actual energy of compartment 2 at $80 \mathrm{~s}$ and the estimated energy of compartment 2 at $80 \mathrm{~s}$ as determined by the identified model are shown. 


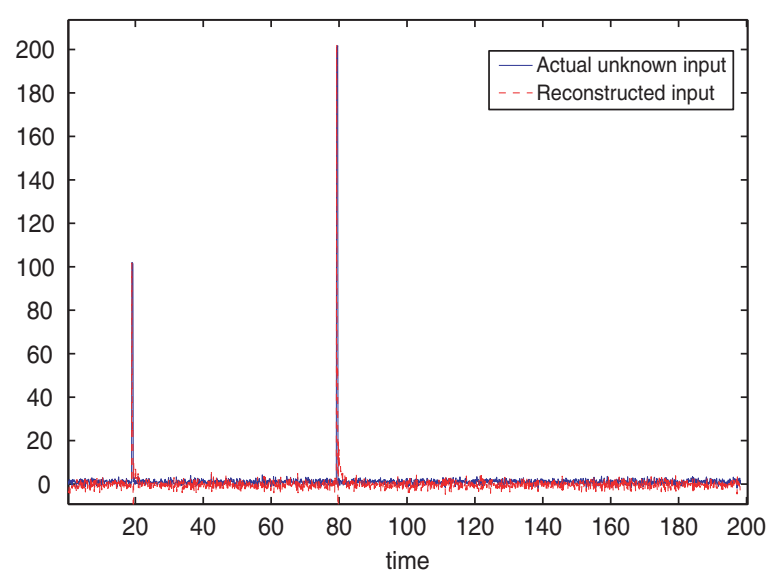

Figure 4. Compartmental model example. The actual unknown input and the estimate of the unknown input are shown.

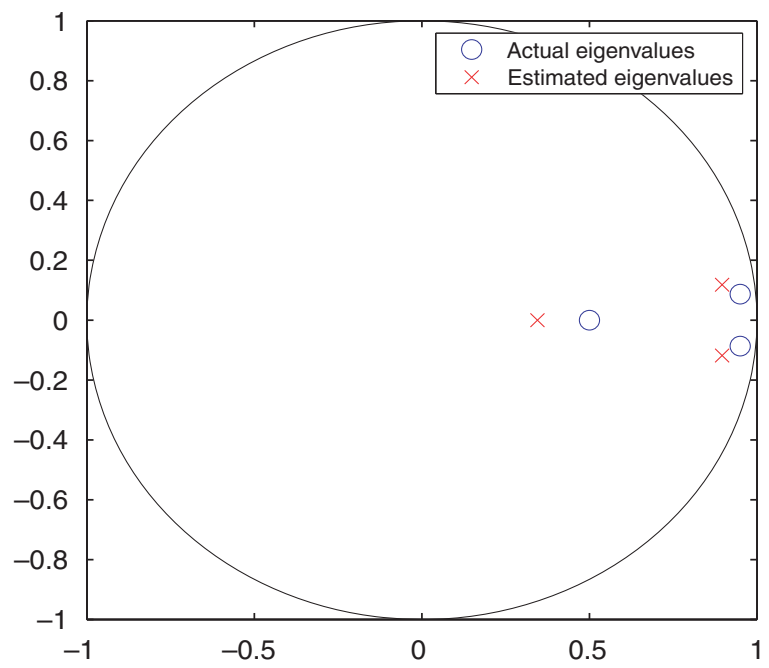

Figure 5. Nonlinear system example. The eigenvalues of $A$ for the linear portion of the system and the eigenvalues of the estimate of $A$ are shown.

\section{NONLINEAR SYSTEM EXAMPLE}

Finally, we consider a system with $n=3$ and an unknown nonlinearity in one of the state equations.

Copyright (C) 2008 John Wiley \& Sons, Ltd.

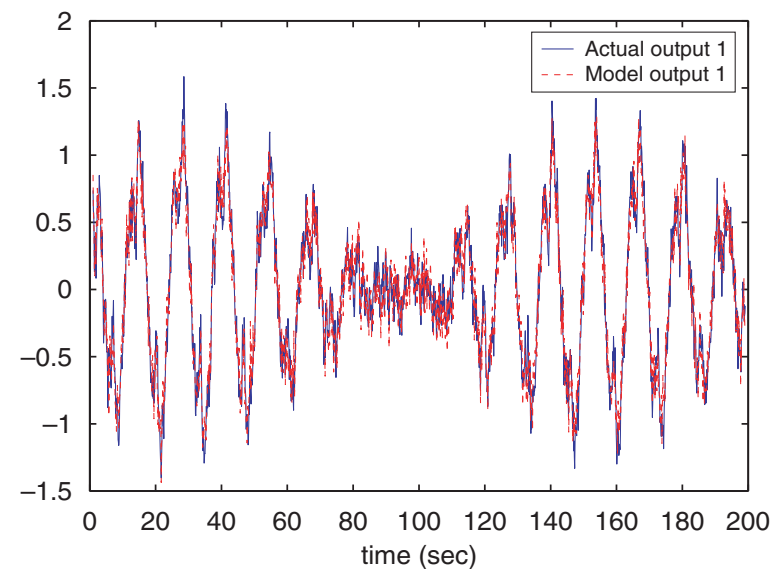

Figure 6. Nonlinear system example. The actual output 1 of the system and the estimated output 1 of the system as determined by the identified model are shown.

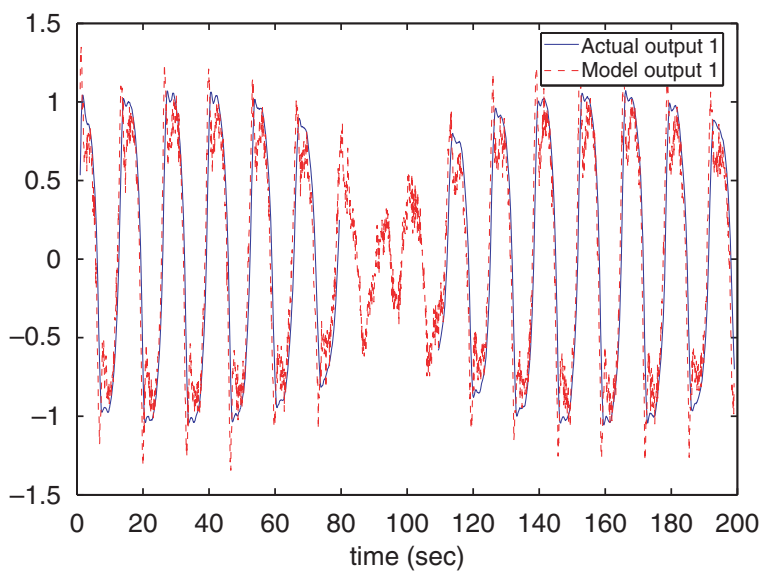

Figure 7. Nonlinear system example. The actual output 2 of the system and the estimated output 2 of the system as determined by the identified model are shown.

Consider the system

$$
\begin{aligned}
x_{1, k+1}= & x_{1, k}+h x_{2, k} \\
x_{2, k+1}= & x_{2, k}+h x_{3, k} \\
x_{3, k+1}= & x_{3, k}-c_{1} x_{1, k}-c_{2} x_{2, k} \\
& -h k x_{3, k}-h x_{1, k}^{3}+u_{k}
\end{aligned}
$$

Int. J. Adapt. Control Signal Process. 2009; 23:1053-1069

DOI: $10.1002 / \mathrm{acs}$ 
where $h$ is the sample interval. We assume that measurements of the first state and the third state are available and the input $u_{k}$ is measured. Thus, the system can be expressed in the form (51), (51) with

$$
\begin{aligned}
A & =\left[\begin{array}{ccc}
1 & t_{s} & 0 \\
0 & 1 & t_{s} \\
-c_{1} & -c_{2} & 1-h k
\end{array}\right], \\
B & =\left[\begin{array}{l}
0 \\
0 \\
1
\end{array}\right], \quad H=\left[\begin{array}{l}
0 \\
0 \\
1
\end{array}\right] \\
C & =\left[\begin{array}{lll}
1 & 0 & 0 \\
0 & 0 & 1
\end{array}\right], \quad D=0, \quad G=0
\end{aligned}
$$

and the unknown signal is the feedback nonlinearity $e_{k}=-h x_{k+1,1}^{3}$. To generate data for identification, we set $k=0.7, c_{1}=0.5, c_{2}=0.6, h=0.1$ and generate 2000 data points with process noise and measurement noise having standard deviation 0.01 . The eigenvalues of the estimate of $A$ are shown in Figure 5, whereas Figures 6 and 7 show the actual outputs of the system and the outputs of the identified model augmented with the nonlinearity identified using (81).

\section{CONCLUSIONS}

In this paper, we considered input and state observability, that is, the ability to estimate both the unknown input and state from the output measurements. We discussed the sufficient and necessary conditions for input and state observability of discrete-time systems. Next, we developed a subspace identification algorithm that identified the state-space matrices and reconstructed the unknown input using output measurements and known inputs. The unknown input could be either an exogenous signal or a nonlinear function of the states. Finally, we presented several illustrative examples.

\section{ACKNOWLEDGEMENTS}

This research was supported in part by the National Science Foundation Information Technology Research initiative, through grant ATM-0325332.

\section{REFERENCES}

1. Emara-Shabaik HE. Filtering of linear systems with unknown inputs. Journal of Dynamic Systems, Measurements, and Control-Transactions of the ASME 2003; 125:482-485.

2. Wang SH, Davison EJ, Dorato P. Observing the states of systems with unmeasurable disturbances. IEEE Transactions on Automatic Control 1975; AC-20:716-717.

3. Hou M, Patton RJ. Input observability and input reconstruction. Automatica 1998; 34(6):789-794.

4. Hou M, Patton RJ. Optimal filtering for systems with unknown inputs. IEEE Transactions on Automatic Control 1998; 43(3):445-449.

5. Kudva P, Viswanadham N, Ramakrishna A. Observers for linear systems with unknown inputs. IEEE Transactions on Automatic Control 1980; 25(1):113-115.

6. Yang F, Wilde RW. Observers for linear systems with unknown inputs. IEEE Transactions on Automatic Control 1988; 33(7):677-681.

7. Kitanidis PK. Unbiased minimum-variance linear state estimation. Automatica 1987; 23(6):775-578.

8. Kolmanovsky I, Sivergina I, Sun J. Simultaneous input and parameter estimation with input observers and setmembership parameter bounding: theory and an automotive application. International Journal of Adaptive Control and Signal Processing 2006; 20:225-246.

9. Kurek JE. The state vector reconstruction for linear systems with unknown inputs. IEEE Transactions on Automatic Control 1983; AC-28(12):1120-1122.

10. Hostetter G, Meditch JS. Observing systems with unmeasurable inputs. IEEE Transactions on Automatic Control 1973; AC-18:307-308.

11. Bhattacharyya SP. Observer design for linear systems with unknown inputs. IEEE Transactions on Automatic Control 1978; AC-23:483-484.

12. Miller RJ, Mukundan R. On designing reduced-order observers for linear time-invariant systems subject to unknown inputs. International Journal of Control 1982; 35(1):183-188.

13. Fairman FW, Mahil SS, Luk L. Disturbance decoupled observer design via singular value decomposition. IEEE Transactions on Automatic Control 1984; AC-29(1):84-86.

14. Kobayashi N, Nakamizo T. An observer design for linear systems with unknown inputs. International Journal of Control 1982; 35(4):605-619.

15. Xiong Y, Saif M. Unknown disturbance inputs estimation based on a state functional observer design. Automatica 2003; 39:1389-1398.

16. Darouach M, Zasadzinski M, Xu SJ. Full-order observers for linear systems with unknown inputs. IEEE Transactions on Automatic Control 1994; 39(3):606-609.

17. Guan Y, Saif M. A novel approach to the design of unknown input observers. IEEE Transactions on Automatic Control 1991; 36(5):632-635.

18. Darouach M. On the novel approach to the design of unknown input observers. IEEE Transactions on Automatic Control 1994; 39(3):698-699.

Int. J. Adapt. Control Signal Process. 2009; 23:1053-1069 
19. Corless M, Tu J. State and input estimation for a class of uncertain systems. Automatica 1998; 34(6):757-764.

20. Glover JD. The linear estimation of completely unknown systems. IEEE Transactions on Automatic Control 1969; AC14(6):766-767.

21. Hautus MLJ. Strong detectability and observers. Linear Algebra and its Applications 1983; 50:353-368.

22. Molinari BP. A strong controllability and observability in linear multivariable control. IEEE Transactions on Automatic Control 1976; 761-764.

23. Sain MK, Massey JL. Invertibility of linear time-invariant dynamical systems. IEEE Transactions on Automatic Control 1969; AC-14(2):141-149.

24. Moylan PJ. Stable inversion of linear systems. IEEE Transactions on Automatic Control 1977; AC-22(1):74-78.

25. Floquet T, Barbot J-P. State and unknown input estimation for linear discrete-time systems. Automatica 2006; 42:1883-1889.

26. Caines PE. Causality, stability and inverse systems. International Journal of Systems Science 1972; 4(5): $825-832$.

27. Bequette BW. Optimal estimation of blood glucose. Proceedings of the IEEE 30th Annual Northeast Bioengineering Conference, Springfield, MA, 2004; 77-78.

28. Van Overschee P, De Moor B. Subspace Identification for Linear Systems: Theory, Implementation, Applications. Kluwer: Dordrecht, 1996.

29. Verhaegen M. Identification of the deterministic part of MIMO state space models given in innovations form from inputoutput data. Automatica 1994; 30(1):61-74.

30. Larimore WE. Canonical variate analysis in identification, filtering and adaptive control. Proceedings of the 29th Conference on Decision and Control, Honolulu, HI, 1990; 596-604.

31. De Cock K, De Moor B. Subspace identification. In Control Systems Robotics and Automation of EOLSS, UNESCO Encyclopedia of Life Support Systems, vol. 1(3), Unbehauen HD (ed.). Eolss Publishers Co., Ltd.:

Copyright (C) 2008 John Wiley \& Sons, Ltd.
Oxford, U.K., 2003; 933-979. Contribution to Section 5.5 (available from: http://www.eolss.net/, ftp://ftp.esat.kuleuven. ac.be/pub/SISTA/goethals/eolss_6_43_9_3.ps).

32. Verdult V. Nonlinear system identification: a state-space approach. Ph.D. Thesis, Faculty of Applied Physics, University of Twente, Enschede, The Netherlands, 2002.

33. Goethals I, Pelckmans K, Suykens JAK, De Moor B. Subspace identification of Hammerstein systems using least squares support vector machines. IEEE Transactions on Automatic Control 2005; 50(10):1509-1519.

34. Van Pelt T, Bernstein DS. Nonlinear system identification using Hammerstein and nonlinear feedback models with piecewise linear static maps. International Journal of Control 2001; 74:1807-1823.

35. Lacy SL, Bernstein DS. Subspace identification of nonlinear systems with measured-input nonlinearities. International Journal of Control 2005; 78(12):906-926.

36. Westwick D, Verhaegen M. Identifying MIMO Wiener systems using subspace model identification methods. Signal Processing 1996; 52:235-258.

37. Lovera M, Gustafsson T, Verhaegen M. Recursive subspace identification of linear and non-linear Wiener state-space models. Automatica 2000; 36:1639-1650.

38. Moonen M, De Moor B, Vandenberghe L, Vandewalle J. On- and off-line identification of linear state space models. International Journal of Control 1989; 49(1):219-232.

39. Moonen M, Vandewalle J. Qsvd approach to on- and offline state space identification. International Journal of Control 1990; 51(5):1136-1146.

40. Bernstein DS, Hyland DC. Compartmental modeling and second-moment analysis of state space systems. SIAM Journal on Matrix Analysis and Applications 1993; 14(3):880-901.

41. Bernstein DS. Matrix Mathematics: Theory, Facts, and Formulas with Application to Linear Systems Theory. Princeton University Press: Princeton, NJ, 2005.

42. Zhou K. Robust and Optimal Control. Prentice-Hall: Upper Saddle River, NJ, 1996. 